\title{
Sulforhodamine Labeling of Neural Circuits Engaged in Motor Pattern Generation in the in vitro Turtle Brainstem-Cerebellum
}

\author{
Joyce Keifer, Devhuti Vyas, ${ }^{a}$ and James C. Houk \\ Department of Physiology, Northwestern University Medical School, Chicago, Illinois 60611
}

\begin{abstract}
A fluorescent molecular probe was used in combination with a novel in vitro preparation to study spatial patterns of neural activity associated with motor pattern generation. The in vitro brainstem-cerebellum preparation takes advantage of the turtle's unusual resistance to anoxia to preserve the entire neural network that connects the cerebellum, red nucleus, and reticular formation. This preparation was bathed in a $0.01 \%$ solution of sulforhodamine while it was activated unilaterally by electrical stimulation of the dorsal quadrant of the spinal cord for $1 \mathrm{hr}$. Sulforhodamine is a small, sulfonated, highly charged fluorescent molecule that is taken up by endocytosis. To examine its distribution in the cerebellum and brainstem, coronal sections were prepared and viewed under epifluorescence illumination. Distinctive spatial patterns of labeling were associated with unilateral electrical stimulation of the in vitro network, suggesting that dye uptake was activity dependent. Blockade of uptake with altered magnesium and calcium concentrations indicated that single spike discharge evoked ortho- or antidromically was insutficient to induce dye uptake. Instead, sulforhodamine staining correlated with the presence of burst discharge that was recorded extracellularly from the red nucleus. Blockade of burst discharge with excitatory amino acid receptor antagonists prevented dye uptake in the red nucleus, the lateral cerebellar nucleus, and other structures that are known to be interconnected by recurrent anatomical pathways. These results suggest that sulforhodamine is internalized by intensely active neurons. The spatial distributions of label support the hypothesis that burst discharges in the turtle red nucleus are mediated by excitatory amino acid neurotransmitters and sustained by recurrent excitation in cerebellorubral synaptic pathways. Positive feedback in these recurrent pathways may provide an important driving force for the generation of motor programs that control limb movements.
\end{abstract}

\footnotetext{
Received Oct. 8, 1991; revised Mar. 13, 1992; accepted Mar. 17, 1992.

We thank Linda Larson-Prior and Kevin McKenna for comments on an earlier version of the manuscript, and Damon LaPorte for photographic assistance. We also thank Tim Skimina and Kevin McKenna for aiding in the development of the confocal imaging system. Tim Skimina and Liang He provided the computer enhanced images in Figure 3. This work was supported by NIH Postdoctoral Fellowship NS 08661 to J.K. and by PHS Grants RO1-NS 21015 and P50MH48185 to J.C.H

Correspondence should be addressed to Dr. Joyce Keifer, Department of Physiology, Northwestern University Medical School, 303 East Chicago Avenue, Chicago, IL 60611.

a Present address: Department of Basic Science and Pharmacology, Memorial University, St. John's, Newfoundland, Canada A1B3V6.

Copyright (c) 1992 Society for Neuroscience $0270-6474 / 92 / 123187-13 \$ 05.00 / 0$
}

Although there has been considerable recent progress in understanding how cellular and network mechanisms in the spinal cord function together in the generation of motor programs that control locomotion (Grillner et al., 1986; Roberts et al., 1986; O'Donovan, 1989), much less is known about how supraspinal systems generate the motor programs that control goal-dirccted movements of the limb. Recently, we developed an in vitro preparation from the turtle in order to explore the cellular and network mechanisms underlying the generation of central motor patterns in cerebellar and red nucleus circuits (Keifer and Houk, 1989 , 1991a). The turtle was chosen because of its unusual resistance to anoxia (Lutz et al., 1985), which permits in vitro studies of extensive neural networks, and also because the neuroanatomical pathways linking the cerebellum, red nucleus, and spinal cord in reptiles are very similar to those found in mammals (Bangma and Ten Donkelaar, 1982; Bangma et al., 1984).

The pathways comprising the reptilian cerebellorubral system are summarized in Figure $1 A$. Reptiles possess a well-developed red nucleus and rubrospinal tract that projects to the contralateral spinal cord (Ten Donkelaar, 1976). The primary input to the red nucleus is from the lateral cerebellar nucleus, homolog of nucleus interpositus, from the oppositc side of the brain (Bangma et al., 1984). While it is likely that the red nucleus receives ascending spinal input (Sarrafizadeh et al., 1990), reptiles lack a motor cortex, and there is no evidence for a descending input from the telencephalon to the red nucleus. Rubrospinal tract axons send collaterals to the lateral cerebellar nucleus and also to neurons in the reticular formation that in turn project to the cerebellar nucleus (Ten Donkelaar, 1982; Bangma et al., 1984), thus forming several recurrent loops. Information carried by these collaterals is also conveyed to the cerebellar cortex in the form of mossy fiber inputs (Kunzle, 1983). Purkinje cells in the cerebellar cortex send a prominent inhibitory projection to the cerebellar nuclei.

The recurrent pathways that link the red nucleus, the reticular formation, and the lateral cerebellar nucleus in reptiles are comparable to recurrent pathways in mammals that have been analyzed with intracellular recording to demonstrate excitatory synapses and the potential for reverberatory activity sustained by positive feedback (Tsukahara et al., 1983). According to a recent theory of sensorimotor integration, positive feedback in these circuits is an important driving force for the generation of limb motor programs (Houk, 1987, 1989). This theory proposes that the burst discharges recorded in the red nucleus in awake cats and monkeys ( $\mathrm{Ghez}$ and Kubota, 1977; Gibson et al., 1985a,b; Cheney et al., 1988), and also in lightly anesthetized turtles (Sarrafizadeh et al., 1989), are expressions of positive feedback in the cerebellorubral circuit. However, it is difficult 


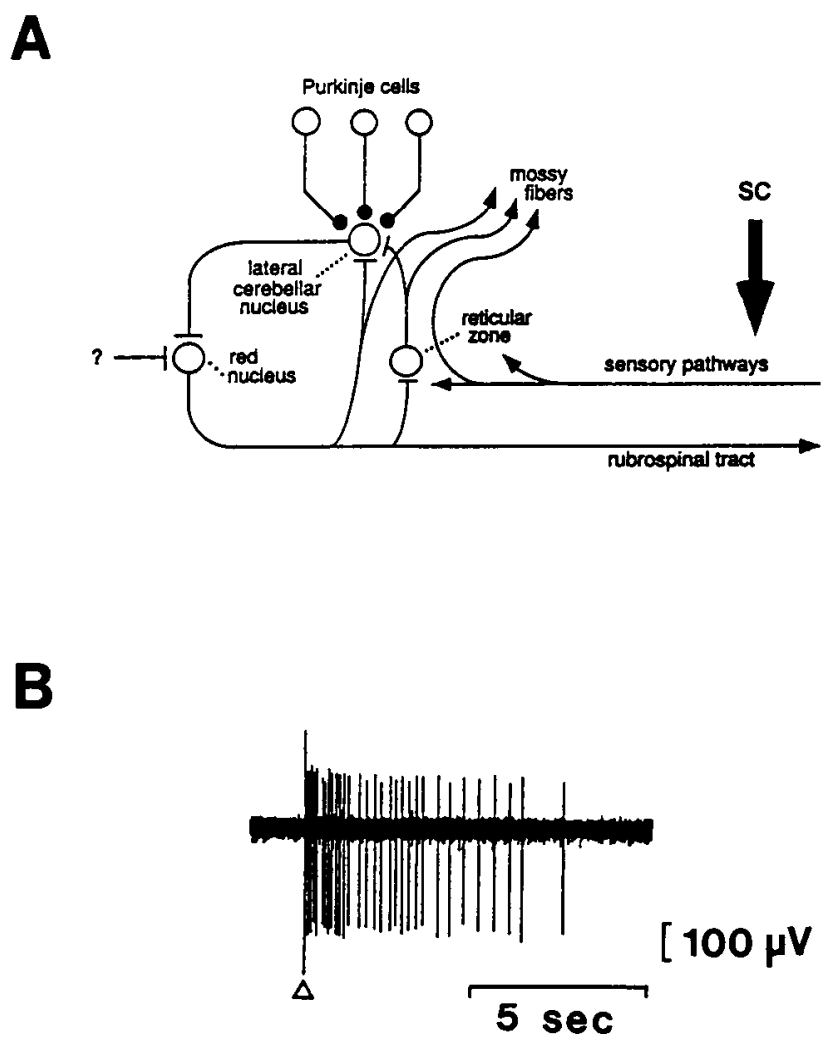

Figure 1. A, Schematic diagram of the cerebellorubral circuit of reptiles. This circuit is very similar to that found in mammals. Neuroanatomical studies demonstrate a number of feedback connections among the cerebellum and brainstem nuclei. In addition, the red nucleus receives indirect sensory input. Whether the turtle red nucleus receives direct input from other sources is not known, and this is indicated by a question mark. In a previous study (Keifer and Houk, 1991a), we hypothesized that bursts of red nucleus discharge $(B)$ recorded in the $i n$ vitro brainstem-cerebellum are triggered by a sensory input and sustaincd by positive feedback among the red nucleus, cerebellar nucleus, and neurons in the reticular formation. The burst shown in $B$ was initiated by a single shock electrical stimulus applied to the contralateral spinal cord $(S C)$.

to study the mechanisms underlying positive feedback and bursting in intact animals.

Burst discharges (Fig. $1 B$ ) can also be recorded from the turtle red nucleus in vitro in response to electrical stimulation of the spinal cord or cerebellum (Keifer and Houk, 1991a). These longduration discharges are in response to brief triggering stimuli and appear to represent central motor patterns analogous to the motor programs recorded from intact animals. The availability of the in vitro preparation has facilitated the study of cellular and circuit mechanisms that contribute to bursting, and, by analogy, to motor program generation. As expected from the circuit shown in Figure 1, bursts are enhanced when inhibitory input to the circuit is interrupted by cutting Purkinje cell axons (Keifer and Houk, 1991 a). Neuropharmacological experiments have further indicated that synaptic transmission in the circuit is mediated, at least in part, by excitatory amino acid receptors of both the NMDA and non-NMDA subtypes, since bursts are blocked by specific antagonists to these receptors (Keifer and Houk, 1991a). It has not yet been possible to record from several sites in this circuit simultaneously to demonstrate that the whole circuit is active during bursting.

In the present study, we sought to use an activity marker to label the neural circuits active during red nucleus bursting. The fluorescent dye sulforhodamine 101 seemed promising since it had been used to label active motor nerve terminals in snake (Lichtman et al., 1985) and epileptiform activity in the turtle visual cortex in vitro (Kriegstein et al., 1988). Sulforhodamine and other small, sulfonated, highly charged fuorescent molecules are used as molecular probes for studying endocytosis and exocytosis (Besterman et al., 1981; Swanson et al., 1985; Wang and Goren, 1987). These molecules do not directly permeate cellular membranes but enter the cell via pinocytotic vesicles. The contents of these vesicles are rapidly cycled into and out of cells, and a fraction of the internalized pool is transferred to lysosomes; molecules such as sulforhodamine and Lucifer yellow accumulate in the lysosomes over a time course of approximately an hour (Swanson et al., 1985; Wang and Goren, 1987). Experiments with peritoneal macrophages demonstrated that the rate and extent of lysosomal accumulation of Lucifer yellow, a molecule in the same class as sulforhodamine, are markedly stimulated by phorbol esters (Swanson et al., 1985). Induced endocytosis of Lucifer yellow was postulated as a likely mechanism for the selective staining of illuminated photoreceptors in the housefly (Wilcox and Franceschiri, 1984). Induced endocytosis could also explain previous reports that sulforhodamine is taken up selectively by neuromuscular junctions that are electrically stimulated (Lichtman et al., 1985) and by nerve terminals at sites of epileptiform activity (Kriegstein et al., 1988), since vesicular turnover at motor nerve terminals is markedly enhanced when neuromuscular transmission is stimulated (Heuser and Reese, 1973).

The present report describes the use of sulforhodamine in the in vitro turtle brainstem-cercbellum preparation. Wc present evidence that dye uptake is dependent on intense synaptic activity, and we describe how this activity is distributed throughout the cerebellorubral circuit under different experimental conditions. Although the spatial patterns of dye uptake are complex, they lend support to the hypothesis that recurrent excitation between the red nucleus and cerebellum is one of the mechanisms that contributes to the generation of motor programs in the cerebellorubral system.

A preliminary account of these experiments was published previously (Houk et al., 1989).

\section{Materials and Methods}

Freshwater pond turtles (Chrysemys picta) of 4-6 inch carapace length were anesthetized by hypothermia $\left(0^{\circ} \mathrm{C}\right.$; Marcus, 1981). Following decapitation, the brain and rostral spinal segments were dissected from the skull in cold physiological saline. The preparation was transferred to the recording chamber, where the brain was transected at the level of the hypothalamus and the telencephalon was discarded. The tissue was pinned down ventral side up and the aqueduct of Sylvius was cannulated with the smooth end of a glass pipette to allow perfusion through the fourth ventricle. The tissue was continuously perfused with physiological saline containing (in $\mathrm{mm}$ ) $100 \mathrm{NaCl}, 6 \mathrm{KCl}, 40 \mathrm{NaHCO}_{3}$, $2.6 \mathrm{CaCl}_{2}, 1.6 \mathrm{MgCl}_{2}$, and 20 glucose, which was oxygenated with $95 \%$ $\mathrm{O}_{2}, 5 \% \mathrm{CO}_{2}$ at room temperature $\left(22-24^{\circ} \mathrm{C}\right)$. Perfusion was discontinued during dye application to the bath.

The in vitro turtle brainstem-cerebellum preparation was bathed in normal physiological saline containing a $0.01 \%$ solution of the dye sulforhodamine 101 (Molecular Probes, S-359) for $1 \mathrm{hr}$ while applying single shock electrical stimuli to the dorsolateral funiculus of the spinal cord with a concentric electrode. This stimulus activates the red nucleus through both synaptic and antidromic pathways (see Fig. $1 A$ ). To ensure that activation of the red nucleus was unilateral, the stimulus was restricted to the dorsolateral funiculus of one side of the spinal cord and the cord was hemisected on the other side. Because the rubrospinal tract in reptiles is completely crossed, this permitted using the red nucleus 
on the opposite side of the brain as a control for nonspecific uptake. In addition, to prevent activation of vestibulospinal axons, the ventral white matter of the cord was cut away horizontally to expose the dorsolateral funiculus. In this way, an attempt was made to activate cerebellorubral pathways as exclusively as possible.

The spinal cord was stimulated once every $2 \mathrm{sec}$ for $1 \mathrm{hr}$ at intensities known to produce burst discharges similar to that shown in Figure $1 B$ in $20-50 \%$ of red nucleus neurons $(4 \mathrm{~mA}, 0.1 \mathrm{msec})$. Stimulation was discontinued, the preparation was washed with physiological saline for $40 \mathrm{~min}$, and the tissue was then immersion fixed in $4 \%$ paraformaldehyde. Transverse sections were cut at $50 \mu \mathrm{m}$ on a freezing microtome, and slides were coverslipped in a 3:1 mixture of glycerol and PBS or antifading mountant $p$-phenylenediamine. Sections were viewed with rhodamine epifluorescence and photographed with Kodak Ektachrome 400. Tissue was stored in the refrigerator or freezer.

Reconstructions of labeled neurons present in the brainstem-cerebellum were made by plotting fluorescently labeled neurons using a drawing tube. Plots were then photoreduced until they matched the size of whole sections drawn with an enlarger. The outline of the section and the plotted neurons were then redrawn into a composite figure, thereby allowing the position of labeled neurons to be reconstructed.

We recently began using a Photometrics cooled CCD camera with a Kodak KAF1400 chip to obtain computerized images of the sections. These images are captured with an aperture scanning confocal microscope, utilizing a slit aperture moving through a conjugate field plane (Lichtman et al., 1989).

To correlate physiological recordings of activity in the red nucleus with dye uptake, burst discharge was recorded extracellularly from single units in the red nucleus during dye application, using methods similar to those described previously (Keifer and Houk, 1991a). Three types of experiments were performed. In some experiments, when brisk burst discharges were recorded from a given neuron, sulforhodamine was applied to the bath. In other cases, burst discharge was pharmacologically blocked during dye application. The excitatory amino acid receptor antagonist CNQX (6-cyano-7-nitroquinoxaline-2,3-dione; $10 \mu \mathrm{M}$ bath application), which blocks the non-NMDA receptor, or APV (DL-2amino-5-phosphonovaleric acid; $100 \mu \mathrm{M}$ bath application), which blocks the NMDA reccptor, was used. These substances were initially bath applied in normal saline until bursting was blocked (usually in $15 \mathrm{~min}$ ). The pharmacological agent was then mixed with sulforhodamine and bath applied for $1 \mathrm{hr}$. These compounds have been previously shown to be potent blockers of activity in the turtle red nucleus (Keifer and Houk, 1991a). Finally, during some experiments, little activity in the red nucleus could be recorded with a number of electrode passes. Sulforhodamine was also applied to these preparations.

\section{Results}

\section{Sulforhodamine uptake during unilateral spinal cord stimulation}

The initial experiments sought to examine the distribution of sulforhodamine uptake in the brainstem-cerebellum preparation that occurred as a consequence of unilateral activation of the ccrcbcllorubral circuit. The results of our previous neurophysiological studies suggested that a single shock $(4 \mathrm{~mA}, 0.1$ $\mathrm{msec}$ ) electrical stimulus applied to the dorsolateral funiculus on one side of the spinal cord elicits burst discharge in $20-50 \%$ of red nucleus neurons contralateral to the side of stimulation in favorable preparations (Keifer and Houk, 1989, 1991a). Using this protocol, the spinal cord was stimulated once every 2 sec for $1 \mathrm{hr}$ while the preparation was bathed in sulforhodamine. After fixation, cutting, and mounting of the tissue, sections were examined under epifluorescence illumination to locate sites of dye uptake.

Label was most frequently seen in cell bodies, although proximal dendrites and nerve terminals were stained at some brainstem and cerebellar sites. Examples of the quality of label of soma and dendritic processes that could be observed under standard epifluorescence viewing are illustrated in Figure 2. Figure $2 A$ shows several large neuronal cell bodies and dendrites that were intensely labeled in the magnocellular reticular nu- cleus. Cellular staining sometimes appeared particulate, which might represent sequestration of the dye into intracellular compartments (Wang and Goren, 1987). Dendritic processes, when labeled, could be followed for hundreds of microns when the focal plane of the image was manipulated. Confocal images obtained with a cooled CCD camera show labeled procesess more clearly, as illustrated for red nucleus neurons in Figure $3 B$. This illustration was obtained by collapsing five consecutive $2-\mu \mathrm{m}$-thick optical sections into a single image. The low-power image of the same section shown in Figure $3 A$ illustrates the unilateral pattern of labeling in response to unilateral stimulation of the spinal cord. Note also that only a fraction of the cells in the red nucleus were labeled. Similarly, the section through the cerebellar cortex in Figure $2 B$ shows well-labeled clusters of Purkinje cells interspersed with areas of unlabeled neurons. It was common that only a fraction of the cells in a labeled nucleus stained with the dye, while many others in the same nucleus showed no evidence of uptake.

The spatial pattern of dye uptake in the brainstem and cerebellum demonstrates a high degree of regional specificity. This is well illustrated by surveying the distribution of label in a series of transverse sections, as shown in Figure 4 (case 2). Note that only a few nuclei contained label, and for the majority of these nuclei staining was present only on one side of the brain. This agreed with the fact that the dorsolateral funiculus on one side of the spinal cord was stimulated. In the cases illustrated in Figures $3 A$ and 4 , stimulation was on the left and resulted in labeling of the right red nucleus (RN in Fig. $4 A$ ) in agreement with the crossed nature of the projections to and from the magnocellular division of the red nucleus. We will refer to dye uptake opposite to the side of stimulation as contralateral labeling.

Label was also present in the ipsilateral nucleus of the medial longitudinal fasciculus (n Flm) and bilaterally, but asymmetrically distributed, in the optic tectum (Fig. 4A). At more caudal levels, label was present in the ipsilateral lateral cerebellar nucleus (Cerl, Fig. 4B), in cerebellar Purkinje cells (PC) bilaterally but with a greater density on the ipsilateral side (Fig. $4 C$ ), and in several zones of the reticular formation, again predominantly ipsilaterally (Fig. $4 D-G$ ). While most of the label appeared in cell bodies, the label shown in Figure $4 F$ is predominantly in nerve terminals, and its ventrolateral position in the caudal brainstem correlates with the expected location of the lateral reticular nucleus (LRN). In mammals, this nucleus receives prominent collateral input from rubrospinal tract axons (Robinson et al., 1987).

Table 1 summarizes the distribution of label found in the five cases included in this first experimental group. Entries to the left in each column indicate the presence ${ }^{* *}$ signifies heavy label, * signifies light label) or absence (0) of labeled neurons contralateral to the side of spinal stimulation; entries to the right signify label present ipsilateral to the stimulus. Some sites in the cerebellorubrai circuit (see Fig. 1) were consistently labeled, whereas at other sites the results were variable. Consistent results were obtained for the lateral cerebellar nucleus (Cerl) showing heavy label on the ipsilateral side and no label on the contralateral side, in agreement with the hypothesis that the spinal stimulation produces unilateral activity in the cerebellorubral circuit. The results in red nucleus (RN) instead were variable. Although label was never present on the unstimulated ipsilateral side (since the red nucleus has crossed projections, the ipsilateral side was unstimulated), labcl was inconsistent on the stimulated contralateral side. Cases 2 and 4 showed heavy label, while case 5 had 


\section{A}

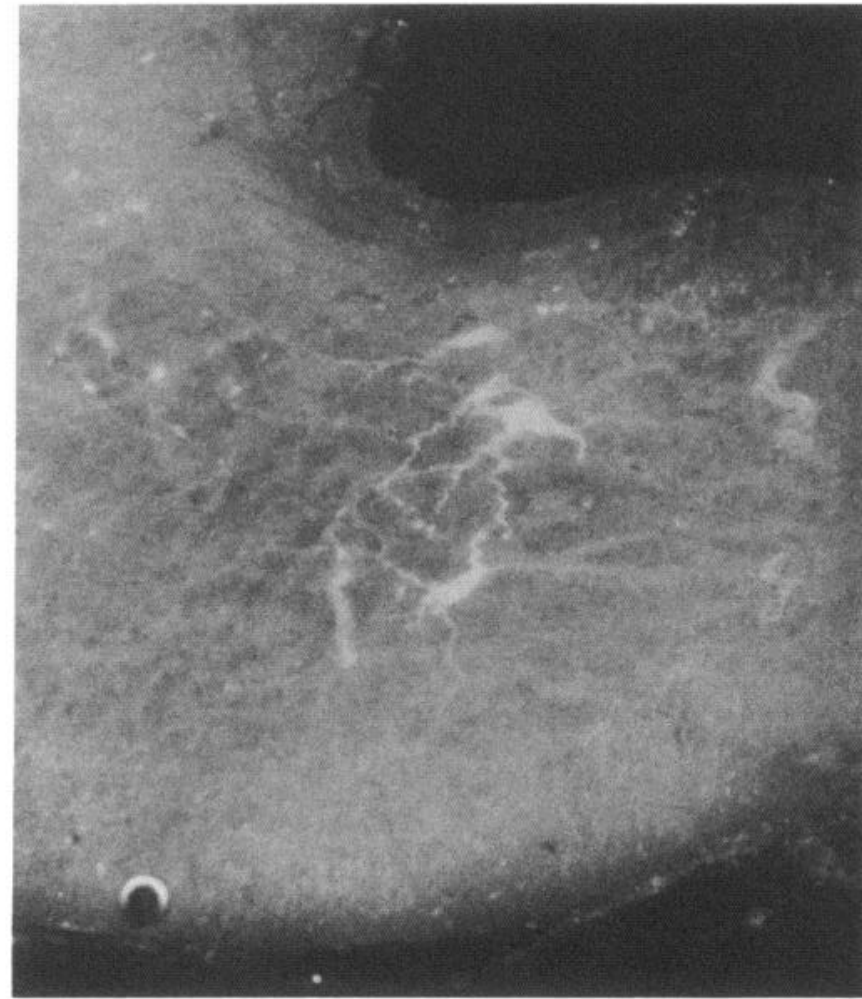

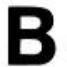

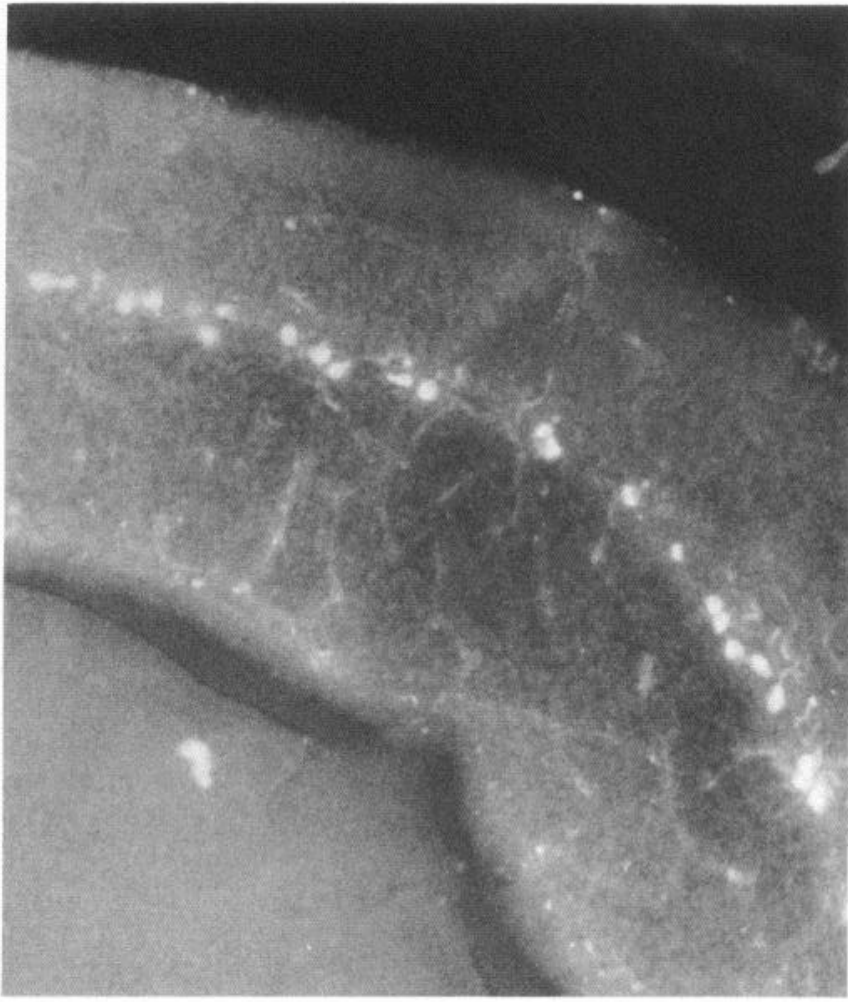

Figure 2. Photomicrographs of selected areas in the turtle brain showing neuronal cell bodies stained with the fluorescent molecule sulforhodamine 101. The in vitro brainstem-cerebellum was activated by spinal stimulation. $A$, Large neurons and dendritic processes are labeled in the ipsilateral magnocellular reticular formation. $B$, Purkinje cells located in the cerebellar cortex stain with the probe. Scale bars, $200 \mu \mathrm{m}$.
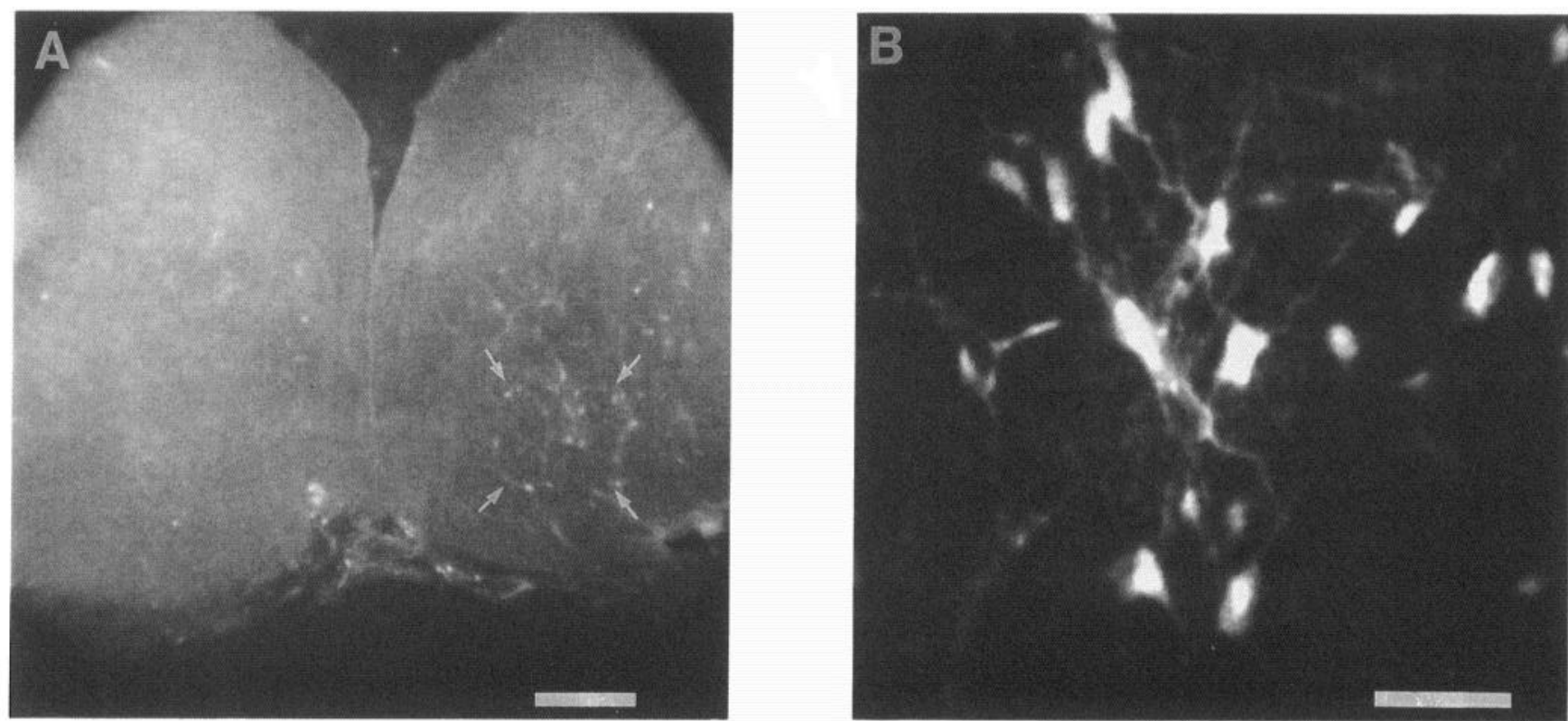

Figure 3. Computer-enhanced images of sulforhodamine-labeled red nucleus neurons taken on an aperture scanning confocal microscope equipped with a cooled CCD camera. $A$, Frontal section through the level of the red nucleus showing a nonconfocal image of labeled red nucleus neurons on the side contralateral to spinal cord stimulation (right, indicated by arrows), while the unstimulated red nucleus (left) shows no label. B, Higherpower confocal image of the same red nucleus neurons as shown in $A$ (right). This photomicrograph was obtained by collapsing five consecutive $2-\mu \mathrm{m}$-thick optical sections into a single image. This procedure results in less out-of-focus light so that fine detail of cell morphology can be resolved. We thank Tim Skimina for providing the images. Scale bars: $A, 250 \mu \mathrm{m} ; B, 100 \mu \mathrm{m}$. 


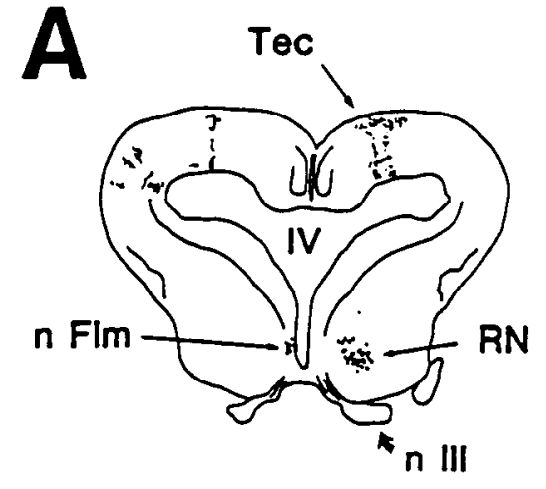

B

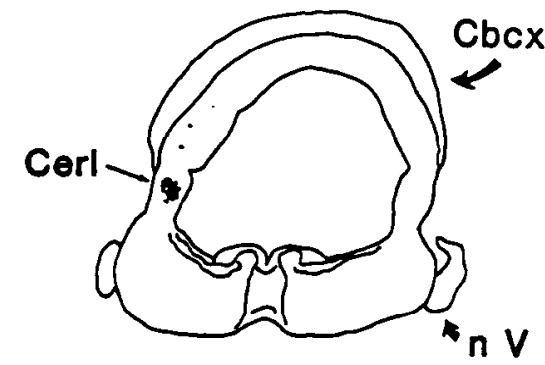

C
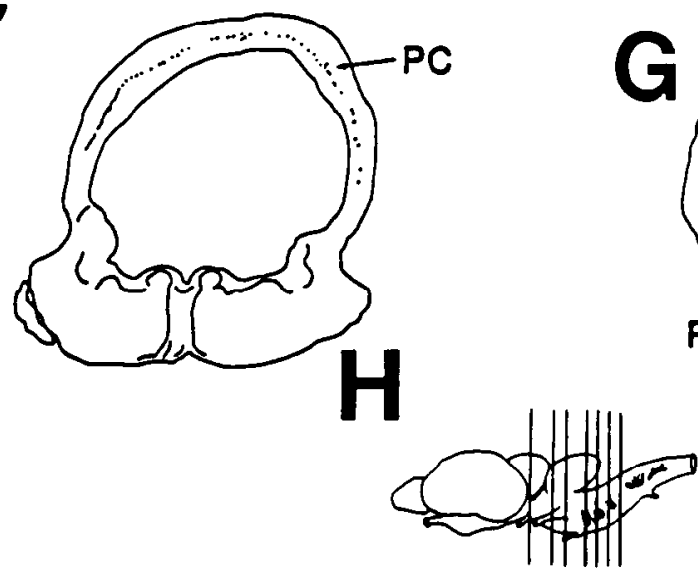

A C EG
D
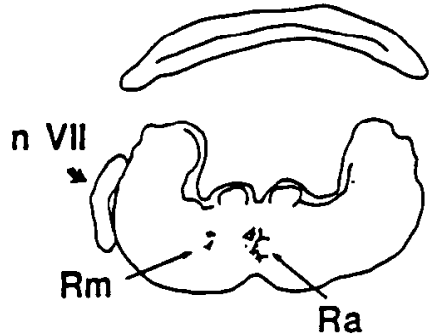

$\mathbf{E}$

$\mathrm{Ra}$

$\mathrm{Rm}$
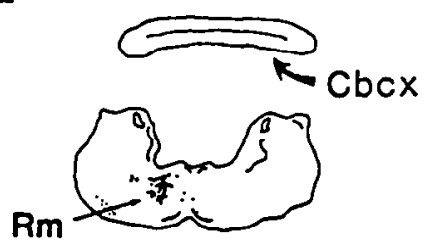

5
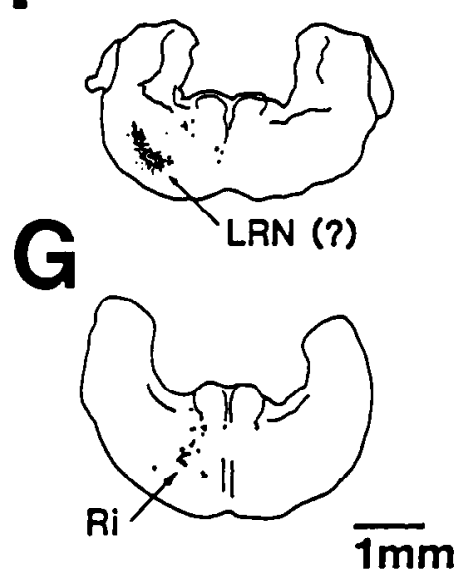

Table 1. Regions in the in vitro turtle brainstem-cerebellum that stain with the dye sulforhodamine 101 following unilateral spinal cord stimulation

Case

\begin{tabular}{|c|c|c|c|c|c|c|c|c|}
\hline number & RN & Cerl & PCs & RF & $\mathrm{Ra}$ & $\mathrm{nFlm}$ & $\mathrm{VeC}$ & Tec \\
\hline 1 & 00 & 0 ** & $* * * *$ & 0 ** & $* *$ & & 00 & -- \\
\hline 2 & $* * 0$ & $* *$ & $* * * *$ & $* * *$ & $* *$ & $0 *$ & 00 & $* *$ \\
\hline 3 & 00 & ** & $0^{* *}$ & $0^{* *}$ & $*$ & $\begin{array}{ll}0 & 0\end{array}$ & ** 0 & $* * * *$ \\
\hline 4 & ** 0 & $* *$ & $0^{* *}$ & $* * *$ & 0 & $0 *$ & $0 *$ & * 0 \\
\hline 5 & * 0 & ** & $* * * *$ & 0 ** & - & $0 *$ & 00 & -- \\
\hline
\end{tabular}

Data to the left in each column are regions contralateral to the site of spinal stimulation; data to the right are ipsilateral to the stimulus. Since the raphe nucleus lies along the midline, it has only one column. ${ }^{* *}$ indicates very intense label: *. light label; 0 , no label present; - , the amount of label was not observed. Abbreviations: $R N$, red nucleus; Cerl, lateral cerebellar nucleus; PCs, Purkinje cells; RF, reticular formation, Ra, raphe nucleus; $\mathrm{nFlm}$, nucleus fasciculus longitudinalis medialis; VeC, vestibular complex; Tec, optic tectum. 
light label and cases 1 and 3 demonstrated no label. In the Purkinje cells of the cerebellar cortex and in the reticular formation, cells were consistently labeled on the stimulated ipsilateral side and label often extended to the contralateral side.

Label was also found in other brainstem structures. Midline reticular neurons in the raphe nucleus $(\mathrm{Ra})$ were labeled in three out of four cases. When present, label in the medial longitudinal fasciculus was ipsilateral as expected from the ipsilateral projections of this nucleus. The vestibular complex (VeC) was labeled in cases three and four as was the ipsilateral medial cerebellar nucleus in case 3 (this nucleus is not included in Table 1 since it rarely labeled). The optic tectum was frequently labeled and in interesting patterns. Typically label was concentrated laterally on the ipsilateral side and medially on the contralateral side as exemplified in Figure 4.

\section{Alterations in uptake produced by lesions of the cerebellorubral circuit}

In a second experimental series, we sought to influence the patterns of dye uptake with lesions of the cerebellorubral circuit. Two preparations with contrasting surgical interventions were studied. In one, the cerebellar nuclei $(\mathrm{CN})$ were lesioned on the ipsilateral side to open the postulated positive feedback loop. This was expected to reduce activity in the red nucleus and at other brainstem sites. In the second type of preparation, the cerebellar peduncle (CP) was cut. An attempt was made to section the entire peduncle; however, invariably the most rostral portion remained intact to prevent damage to the cerebellar nuclei. This lesion transects most Purkinje cell axons as they course to the lateral cerebellar nucleus and removes their inhibitory inputs (see Fig. 1). Previously, we reported that CP lesions result in increased spontaneous and burst activity in the red nucleus (Keifer and Houk, 1991a). Usually two preparations, one of each type, were studied simultaneously in the same bath.

When the cerebellar nuclei were lesioned, there was no dye uptake in the red nucleus (cases 6-8 in Table 2). This was expected since the lesion interrupts the cerebellorubral recurrent loop and eliminates the main excitatory input to the red nucleus. We had anticipated that the contrasting lesion produced by cutting the cerebellar peduncle would ensure dye uptake in the red nucleus (cases 9-11 in Table 2). Instead, the CP lesion had a minimal effect when compared with the unlesioned results reported in the previous section. The appreciable dye uptake in the lateral cerebellar nucleus was also similar to our results in the unlesioned preparation and ensured that the CP lesion had not damaged the cerebellar nuclei. Case 12 , a control with the cerebellar peduncle cut but with no spinal stimulation, gave the result of no dye uptake in this presumed quiescent preparation.

The distribution of label throughout the brainstem and cerebellum is shown for a CN lesion case in Figure 5 (case 6), and for a CP lesion case in Figure 6 (case 9). The data for all the lesioned preparations are summarized in Table 2 . The reticular formation was heavily labeled in all three cases with CP lesions and in two of three cases with $\mathrm{CN}$ lesions, although the exact regions labeled differed in the two types of preparation. With the C.P lesion, the label was concentrated in the magnocellular and inferior reticular formation ( $\mathrm{Rm}$ and $\mathrm{Ri}$ in Fig. 6), which is similar to the location of label in the unlesioned cases (Fig. 4). However, with the $C N$ lesion, label was shifted to more lateral and rostral sites in the superior reticular formation (Rs in Fig. 5) rather than in the magnocellular reticular formation. The raphe nucleus was labeled in both $\mathrm{CP}$ and $\mathrm{CN}$ cases, though it was most intense and consistent in the CP lesion cases. Purkinje cells in the CP lesion cases were also found to be intensely labeled and predominantly on the ipsilateral side.

\section{Specific tests of activity dependence}

The results presented in the previous sections are consistent with the hypothesis that the uptake of sulforhodamine is in some way related to neuronal activity. Nonetheless, the results were too complex and too variable to draw any firm conclusions. The experiments described in the present section were designed to explore further the dependence of uptake on the type and intensity of neuronal activity.

First, we sought to determine whether or not antidromic action potentials are sufficient to cause cells to take up the dye, or whether some form of synaptic activation is instead required. The in vitro preparation was bathed in low-calcium $(0.1 \times$ normal), high-magnesium $(2 \times$ normal) solution, a medium that has been used to block synaptic transmission while not interfering with the propagation of action potentials (Keifer and Houk, 1989). The results (cases 13 and 14 in Table 3) were a total absence of dye uptake in the brainstem. In case 14, a small cluster of Purkinje cells was stained, raising the possibility that endogenous bursting activity such as can be recorded from these neurons (Chan and Nicholson, 1986) may be sufficient to cause uptake. This result also shows that it is possible for the dye to be taken up in the low-calcium medium.

One way to reduce synaptic activity without completely abolishing it is to bath the preparation in a solution containing high calcium $(2 \times$ normal) and high magnesium $(2 \times$ normal). Such a solution has been shown to block polysynaptic transmission without affecting monosynaptic transmission (Berry and Pen-

Table 2. Alterations in uptake of sulforhodamine 101 by lesions

\begin{tabular}{|c|c|c|c|c|c|c|c|c|c|c|c|c|c|c|}
\hline $\begin{array}{l}\text { Case } \\
\text { number }\end{array}$ & Manipulation & RN & Cer & & $\mathbf{P C}$ & & $\mathbf{R}$ & & $\mathrm{Ra}$ & nFlm & $\mathrm{V}$ & $\mathrm{CC}$ & Tec & \\
\hline 6 & $\mathrm{CN}$ lesion & 00 & - & - & 0 & * & 0 & $* *$ & $*$ & 00 & 0 & * & $* *$ & ** \\
\hline 7 & $\mathrm{CN}$ lesion & 00 & - & - & 0 & * & 0 & 0 & $*$ & 00 & 0 & $* *$ & 0 & 0 \\
\hline 8 & $\mathrm{CN}$ lesion & 00 & - & - & 0 & 0 & $*$ & $* *$ & $*$ & 00 & $*$ & ** & 0 & 0 \\
\hline 9 & Cut CP & $* * 0$ & 0 & * & $*$ & ** & $*$ & $* *$ & $* *$ & 00 & 0 & * & $*$ & 0 \\
\hline 10 & Cut CP & * 0 & 0 & ** & * & ** & 0 & $* *$ & $* *$ & 00 & 0 & 0 & - & - \\
\hline 11 & Cut CP & 00 & 0 & $* *$ & 0 & $* *$ & 0 & $* *$ & $* *$ & 00 & 0 & 0 & 0 & 0 \\
\hline 12 & Cut $C P$, no stimulation & 00 & 0 & 0 & 0 & 0 & 0 & 0 & 0 & 00 & 0 & 0 & 0 & 0 \\
\hline
\end{tabular}

See Table 1 notes for explanation of format and abbreviations. 
A
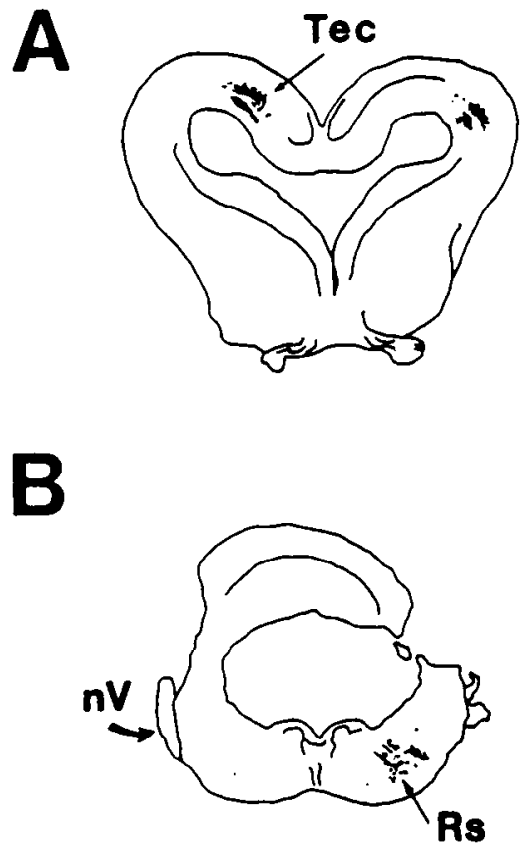

0

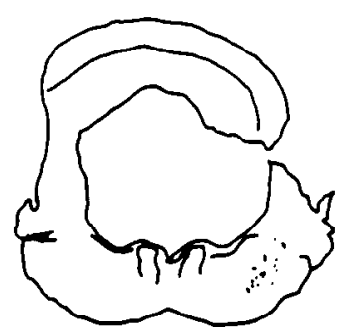

D

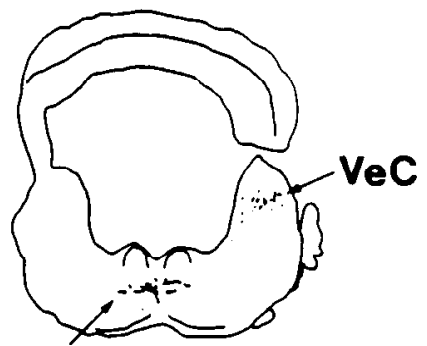

Ra
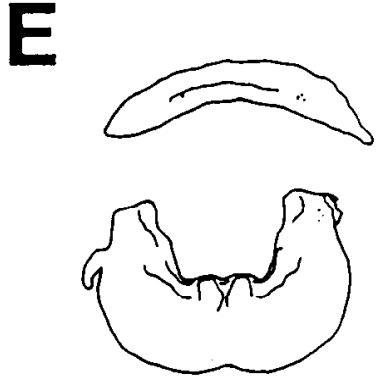

F

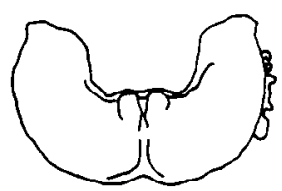

6

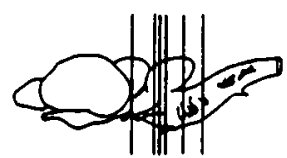

Figure 5. Pattern of sulforhodamine uptake after the cerebellar nuclei were lesioned on one side of the brain (case 6). This preparation was placed in the bath containing sulforhodamine simultaneously with the preparation shown in Figure 6, which received a contrasting lesion. This lesion, shown in $B$ and $C$. interrupts the recurrent loops via the lateral cerebellar nucleus to the red nucleus (Fig. 1). Ipsilateral to the site of spinal cord stimulation is to the right. This surgical intervention resulted in very little uptake of sulforhodamine compared to the case shown in Figure 6 . No neurons in the red nucleus were labeled $(A)$. However, layers in the optic tectum were labeled laterally on the ipsilateral side and medially on the contralateral side $(A)$. A cluster of superior reticular $(R s)$ neurons were stained ipsilateral $(B$ and $C$ ), as were a few raphe neurons and cells in the vestibular complex $(\mathrm{VeC}, \mathrm{D})$. Other abbreviations and format are as in Figure 4. treath, 1976). In a previous study, we reported that burst discharge in red nucleus is blocked in this solution, even though some red nucleus neurons can still be synaptically activated to discharge a single action potential in response to stimulation of the spinal cord (see Fig. 5 in Keifer and Houk, 1991a). As illustrated by case 15 in Table 3, when the preparation was treated in this manner, there was very little uptake; only a few raphe neurons and Purkinje cells were labeled. It appeared that single action potentials evoked at a low frequency, even when synaptically driven, are insufficient for dye uptake. Intense synaptic activity may be a critical feature in labeling neurons with sulforhodamine.

To test this idea, we attempted to correlate dye uptake with the degree of bursting activity, as assessed by extracellular mi-

Table 3. Tests of activity dependence of uptake with physiological recording, and ionic and pharmacologic blockade

\begin{tabular}{|c|c|c|c|c|c|c|c|c|c|c|c|}
\hline $\begin{array}{l}\text { Case } \\
\text { number }\end{array}$ & Manipulation & RN & Cerl & $\mathrm{PCs}$ & RF & $\mathbf{R a}$ & $\mathrm{nFlm}$ & $\mathrm{VeC}$ & & Tec & \\
\hline 13 & Low $\mathrm{Ca}$, high $\mathrm{Mg}$ & 00 & 0 & 00 & 0 & 0 & 00 & 0 & 0 & 0 & 0 \\
\hline 14 & Low $\mathrm{Ca}$, high $\mathrm{Mg}$ & 00 & 00 & $0 *$ & 0 & 0 & 00 & 0 & 0 & 0 & 0 \\
\hline 15 & High $\mathrm{Ca}$, high $\mathrm{Mg}$ & 00 & $0 \quad 0$ & $*$ & 0 & * & 00 & 0 & 0 & 0 & 0 \\
\hline 16 & Burst discharge & $* * 0$ & $0^{* *}$ & $0 *$ & $0 *$ & $*$ & 00 & 0 & 0 & $* *$ & $* *$ \\
\hline 17 & Burst discharge & $* 0$ & $0 *$ & * ** & $*$ & $*$ & $0^{*}$ & * & 0 & $* *$ & 0 \\
\hline 18 & No burst discharge & $* * 0$ & 0 & 00 & $0 *$ & $*$ & 00 & 0 & $* *$ & 0 & 0 \\
\hline 19 & No burst discharge & 00 & 0 & $*$ & $* * *$ & $*$ & 00 & $*$ & $*$ & 0 & 0 \\
\hline 20 & CNQX & 00 & 0 & 0 & $0 * *$ & $*$ & 00 & $* *$ & $* *$ & 0 & ** \\
\hline 21 & CNQX & 00 & 0 & 0 & $0 *$ & $*$ & 00 & 0 & 0 & 0 & 0 \\
\hline 22 & APV & 00 & 0 & 0 & 0 & 0 & $0 *$ & 0 & * & $* *$ & ** \\
\hline
\end{tabular}


Figure 6. Pattern of sulforhodamine uptake after the cerebellar peduncle had been cut unilaterally $(C$ and $D)$. This lesion (case 9 ) interrupted the pathway by which Purkinje cells inhibit the lateral cerebellar nuclei. Ipsilateral to the site of spinal cord stimulation is to the right. In this experiment, the contralateral red nucleus is well labeled $(A)$, as is the ipsilateral lateral cerebellar nucleus $(B)$. Purkinje cells $(B-D)$, raphe $(C)$, and some magnocellular reticular neurons $(C-E)$ are stained bilaterally. A few neurons in the ipsilateral vestibular complex also were labeled $(\mathrm{VeC}$, $D$ ). Unlike the other cases, labeled cells in the optic tectum were positioned laterally on the contralateral side. Other abbreviations and format are as in Figure 4.

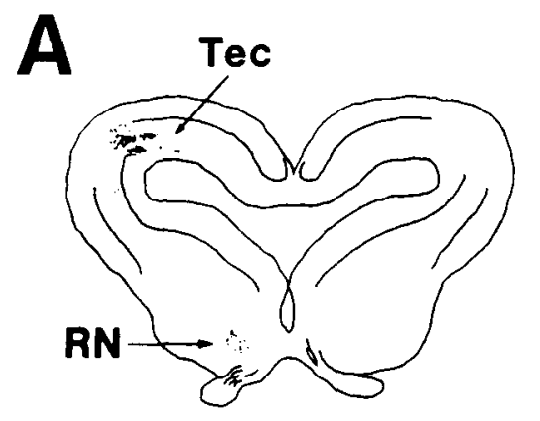

B
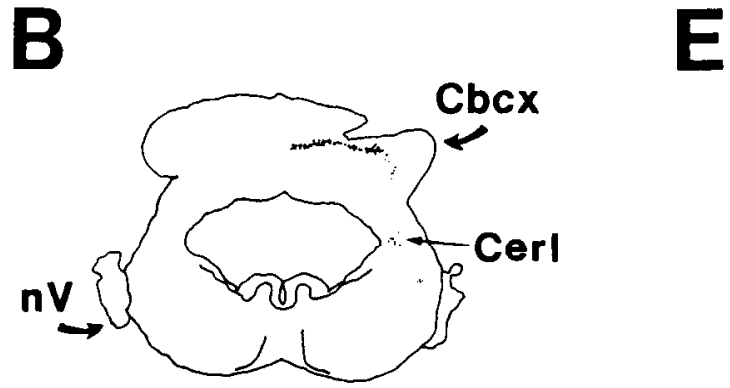

D
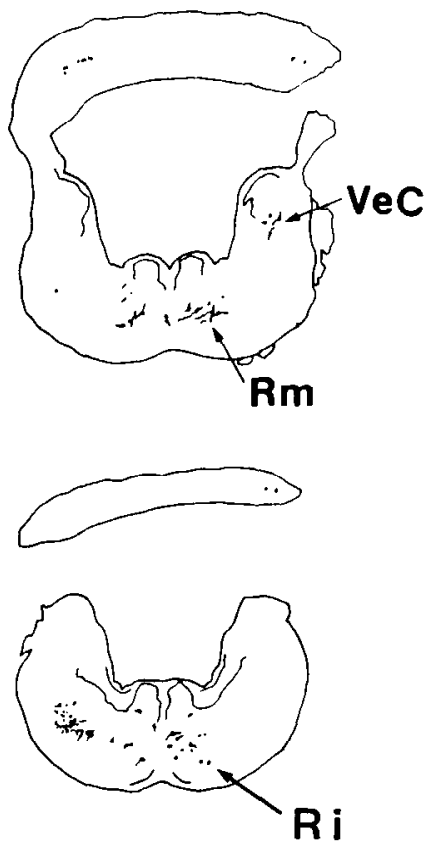

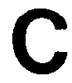
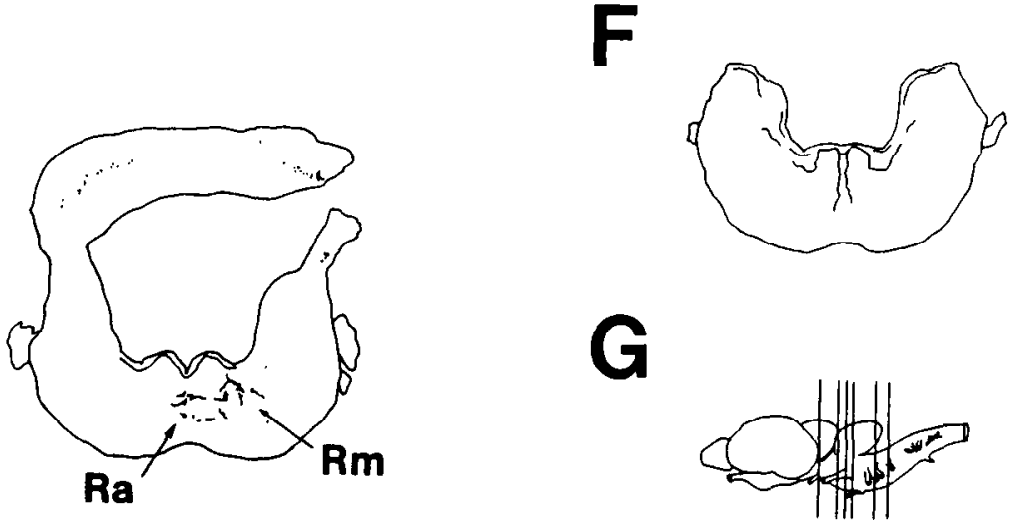

croelectrode recordings, in the red nucleus. In cases 16 and 17 , vigorous burst activity in response to spinal stimulation was recorded from the red nucleus prior to the addition of sulforhodamine, and the contralateral red nucleus was labeled (Fig. $7 B$, Table 3). In two other cases, bursting was not recorded from any units although numerous penetrations were made through the red nucleus. There was no red nucleus label in case 19; however, many red nucleus cells were intensely labeled in case 18 . We do not know why cells were labeled in case 18 , though we suspect that it might be related to injury produced by the large number of penetrations (10) that were made through the red nucleus. Consistent with this interpretation, we found label in the red nucleus in an unstimulated control preparation in which multiple penetrations were made through the nucleus.

Previous results have shown that the excitatory amino acid receptor antagonists CNQX and APV are potent blockers of burst discharge in the red nucleus (Keifer and Houk, 1991a). Use of these antagonists provided another means for testing whether dye uptake depended on burst discharge. Microelectrode recordings from the red nucleus ensured that burst activity was initially present and that it was blocked by the pharma- cological agent prior to and during the application of the dye. Figure $7 A$ (Table 3, case 20) shows a recording from a red nucleus neuron in which burst discharge had been blocked by CNQX during application of the dye. No label was observed in the red nucleus. Similar results were obtained with the application of APV. Data presented in Table 3 for cases 20-22 show a strong correlation between the level of activity in the red nucleus and the amount of label observed there, though there was uptake at other (neurophysiologically unmonitored) sites.

Previous studies reported that the uptake of another vital dye, 4-Di-2-Asp [4(4-diethylaminostryl)- $N$-methylpyridinium iodide; Molecular Probes, D-289], in neural tissue is not activity dependent (Lichtman et al., 1989). Consistent with these findings, we found that soaking the isolated brainstem-cerebellum in a $0.01 \%$ solution of this dye resulted in a bilaterally homogeneous pattern that appeared to label myelin rather than cell bodies. The same quality of staining occurred without electrical stimulation of the preparation, or in a bathing solution containing low calcium. This dye was visualized using fluorescein optics and was found to be useful as a "counterstain" when used sequentially with sulforhodamine. 


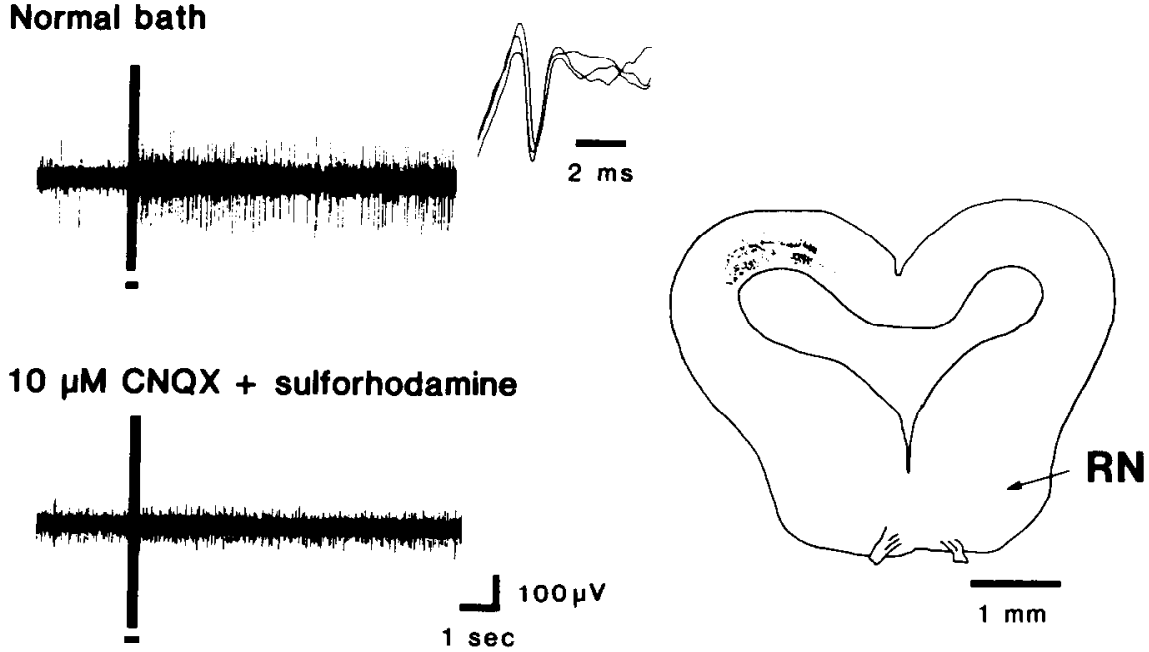

Normal bath + sulforhodamine

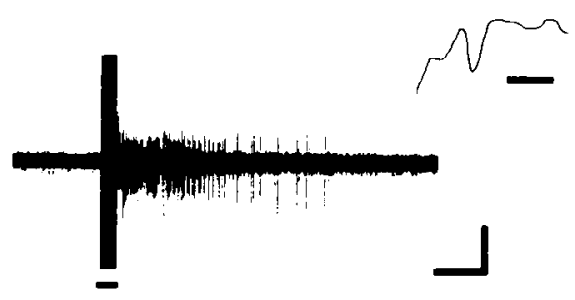

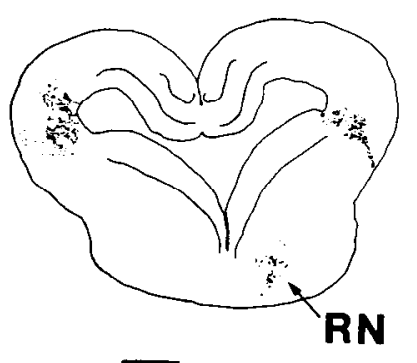

Figure 7. The activity of single neurons recorded extracellularly in the red nucleus during application of sulforhodamine correlates with the presence of label in the red nucleus. $A$, In normal bathing solution, a cell (case 20) that responded to spinal cord stimulation with a latency of $5 \mathrm{msec}$ also produced burst discharges in response to a train stimulus applied to the cord (indicated by the bar). Once the bath application of $10 \mu \mathrm{M}$ CNQX blocked the activity of this cell (usually 15-20 min), sulforhodamine mixed with $10 \mu \mathrm{M}$ CNQX was added to the bath for $1 \mathrm{hr}$ using the same protocol for uptake as described for all the other cases. The section to the right illustrates that no label was found in the red nucleus $(R N)$ under these conditions. The ipsilateral tectum did take up the dye. $B$, Unit (case 16) that responded in $4 \mathrm{msec}$ to cord stimulation also produced burst activity following a train stimulus to the cord. Sulforhodamine was added to the normal bath solution while recording activity of this cell. The section shown to the right indicates that the red nucleus was well labeled in this case.

\section{Discussion}

The distinctive spatial patterns of sulforhodamine uptake found in these experiments provide valuable information for understanding how motor programs may be generated by the cerebellum and brainstem. Before discussing that topic, however, we will evaluate the extent to which sulforhodamine uptake can be correlated with sites of neuronal activity.

\section{Sulforhodamine uptake}

The asymmetry observed in the distribution of label in the cerebellorubral circuit provides important support for the hypothesis that sulforhodamine uptake is activity dependent. The placement of the spinal stimulating electrode in a surgically isolated dorsal quadrant of the spinal cord should have restricted cerebellorubral circuit activity to selected pathways on one side of the brain. Therefore, the absence of label on the nonactivated side provides an important control for evaluating activity-dependent uptake. The consistent labeling of the lateral cerebellar nucleus and the reticular formation on the side ipsilateral to the spinal stimulation strongly supports an activity-dependent mechanism, since these nuclei have an ipsilateral organization.

The red nucleus instead has a contralateral organization. Therefore, we expected to see contralateral staining of this nucleus, which was found in the majority of cases. However, in other cases the contralateral red nucleus remained unlabeled.
This variability in labeling is probably the result of differences in the incidence of burst discharge in red nucleus. The experiments in which recordings were made from the red nucleus before and during the application of sulforhodamine support this interpretation. Label was present in both cases in which bursting was recorded. However, the presence of label in one of the cases in which bursting was not recorded suggests that additional factors may be involved. Our interpretation is that this label resulted from neuronal damage caused by the multiple electrode penetrations made through the nucleus. Red nucleus staining was not seen in unstimulated controls in which no recording was attempted, nor was it seen when burst activity was blocked pharmacologically and recordings were made after only one or two electrode penetrations, whereas staining was present in an unstimulated control in which multiple electrode penetrations were made through the red nuclcus. Furthermore, tissue near the borders of the preparation that was inadvertently damaged in the dissection occasionally took up the label.

The cases involving changes in ionic composition and pharmacologic blocking agents are particularly useful in evaluating possible mechanisms of sulforhodamine uptake. Although the low-calcium/high-magnesium solution blocks synaptic transmission, it should not have interfered with antidromic activity in the red nucleus (Keifer and Houk, 1989). The absence of label in these cases implies that synaptic activity is required to stimulate uptake. The absence of red nucleus staining with the high- 
calcium/high-magnesium solution further suggests that the synaptic activity must be intense. This solution does not prevent the synaptic driving of a single action potential in the red nucleus but instead blocks burst discharge attributed to intense and sustained synaptic activity (Keifer and Houk, 1991a). The results with CNQX and APV substantiate this interpretation, since recordings from the red nucleus during drug application demonstrated that burst activity was indeed blocked before and during the exposure of the tissue to sulforhodamine.

Previous reports that sulforhodamine is taken up selectively by neuromuscular junctions that are activated (Lichtman et al., 1985) and by nerve terminals at sites of epileptiform activity (Kriegstein et al., 1988) fit well with the idea that uptake is an activity-dependent endocytotic process, since vesicular turnover at motor ncrve terminals is markedly enhanced when neuromuscular transmission is stimulated (Heuser and Reese, 1973). In the present experiments, we found label in some nerve terminals, but most of the label was present in cell bodies and dendrites. Often the dye was not distributed uniformly through the cytoplasm but instead assumed a granulated appearance typical of containment in membrane-bound compartments. The dominance of cellular label might have resulted from presynaptic uptake of sulforhodamine at nerve endings followed by rapid axoplasmic transport to the cell bodies. Alternatively, sulforhodamine might be taken up at somatic or dendritic synapses. Dendritic uptake of other substances was demonstrated to occur in Purkinje cells where transport of label from dendrites to soma proceeds with a time course (30-90 $\mathrm{min}$ ) appropriate to explain our results (Borges et al., 1985). Sulforhodamine experiments with shorter exposure and/or wash times might help to clarify these issues.

A specific link between neural activity and endocytotic uptake is unclear at present. The correlation observed between red nucleus bursting and sulforhodamine labeling suggests that intense synaptic activity is required. Judging from the parallel effects of CNQX and APV on neural discharge and dye uptake, excitatory amino acids are likely neurotransmitter candidates at many of these synapses. Metabotropic receptors for excitatory amino acid transmitters are known to promote the postsynaptic formation of diacylglycerol (Sladeczek et al., 1988). Diacylglycerol might induce endocytosis as phorbol ester analogs do in macrophages (Swanson et al., 1985).

In summary, the present results are consistent with the hypothesis that the uptake of sulforhodamine is dependent on activity-related endocytosis. However, we do not know if this occurs mainly at presynaptic nerve terminals, or if uptake in dendrites and soma predominates under the conditions of the present experiments. Furthermore, it appears that intense synaptic activity may be required to label neurons, though we do not yet know what aspect of neural activity is critical, or precisely how this leads to the induction of endocytosis.

\section{Analysis of labeling patterns in the cerebellorubral circuit}

In the present experiments, excitation of the cerebellorubral circuit, and other brainstem circuits, was produced by unilateral, single-shock stimulation of the spinal cord. The existence of a sensory pathway via the spinal cord to the red nucleus is assured by the somatosensory responses reported for intact turtles (Sarrafizadeh et al., 1990). Anatomical studies in turtles and other reptiles have shown direct ascending projections to areas in the reticular formation, cerebellar cortex, raphe nucleus, and optic tectum, but no direct connections to the red nucleus (Ebbesson,
1969; Kunzle and Woodson, 1982). The rubral burst discharge recorded in response to spinal stimulation may be mediated via transmission through one or more of these nuclei, all of which were labeled by sulforhodamine uptake. The cerebellorubral circuit might also have been activated via collaterals of antidromically stimulated rubrospinal fibers. These collaterals synapse with cells in the lateral cerebellar nucleus and reticular formation, as illustrated in Figure 1.

After red nucleus discharge is initiated by an ascending input, some mechanism must sustain burst discharge for the severalsecond durations observed in extracellular recordings. One possibility is that red nucleus cells have endogenous bursting properties, though intracellular studies have failed to demonstrate ion currents that would be capable of sustaining this burst discharge (Kubota et al., 1985; Keifer and Houk, 1991 b). Another possibility is that activity spreads to other neurons in the anatomically demonstrated recurrent pathways that connect the red nucleus, reticular formation, and cerebellum (Fig. 1; cf. introductory remarks). Burst discharge might then be sustained by regenerative positive feedback in these recurrent loops. A third, and most likely, possibility is that a combination of cellular and circuit properties operate in a synergistic fashion to sustain burst discharge (Keifer and Houk, 1991 a). The patterns of dye uptake seen in the present experiments help to define the extent of the cerebellorubral circuit that may become engaged in sustaining burst discharge.

We used a variety of methods to demonstrate that sulforhodamine uptake in the lateral cerebellar nucleus and the reticular formation is a necessary, though not sufficient, condition for observing uptake in the red nucleus. This correlation is confirmed for the normal preparation by cases 2,4 , and 5 in Table 1 , in CP lesion preparations by cases 9 and 10 in Table 2, and in preparations where bursting discharge was monitored physiologically by cases 16 and 17 in Table 3 . The sole case in which red nucleus uptake was not accompanied by lateral cerebellar nucleus uptake (case 18) is attributable to the damage-induced uptake discussed earlier. Additional results indicate that red nucleus uptake does not occur when the cerebellorubral loop is interrupted by interfering with activity in the lateral cerebellar nucleus. This was shown most directly by the three $\mathrm{CN}$ lesion cases in Table 2 . It is also shown by the cases in which ionic substitution or pharmacological agents blocked uptake in the lateral cerebellar nucleus (cases $13-15$ and $20-22$ in Table 3). Finally, the uptake patterns seen in cases 1,3 , and 11 suggest that the presence of activity in the lateral cerebellar nucleus is not sufficient to ensure red nucleus uptake.

That the lateral cerebellar nucleus can in some cases be labeled in the absence of red nucleus label requires further discussion. First, an explanation is needed for the absence of label in the red nucleus under conditions in which the lateral cerebellar nucleus is active; this could be explained if mechanisms existed for the selective inhibition of the cerebellorubral pathway. One of several possibilities is that inhibitory interneurons in the red nucleus (Keifer et al., 1992) were particularly responsive in these preparations. Second, an explanation is needed for the presence of uptake in the lateral cerebellar nucleus in the absence of red nucleus label; one might expect this to interrupt an important source of recurrent excitation. One possible explanation is that activity in the lateral cerebellar nucleus is not strictly dependent upon recurrent excitation. Activity could also result from intense afferent input to the nucleus from some nonrecurrent source, or it could result from the rebound bursting reported to occur 
in cerebellar nuclear cells (Jahnsen, 1986; Llinas and Muhlethaler, 1988). A third possibility is that the anatomy summarized in Figure 1 may not include all of the recurrent connections that actually return to the lateral cerebellar nucleus. In particular, there is a direct recurrent pathway demonstrated in mammals between the interpositus and the nucleus reticularis tegmenti pontis (Tsukahara et al., 1973, 1983). The labeling seen in the reticular formation in cases 1,3 , and 11 is consistent with a reticular loop providing recurrent excitation to the lateral cerebellar nucleus. Further studies are needed to evaluate these alternative possibilities.

The patterns of Purkinje cell label are also important to analyze. Ipsilateral Purkinje cells send their axons to the ipsilateral cerebellar nucleus where they would be expected to inhibit activity (Fig. 1). In spite of the inhibitory nature of this projection, uptake by ipsilateral Purkinje cells consistently occurred in combination with ipsilateral uptake in the lateral cerebellar nucleus. However, it is important to point out that not all of the Purkinje cells were labeled. Some patches of cells were heavily labeled while the majority remained unlabeled. Similarly, patches of labeled neurons were interspersed with patches of unlabeled neurons in the red nucleus (Fig. $3 A$ ), reticular formation, and cerebellar nuclei. In previous physiological studies of bursting in the red nucleus (Keifer and Houk, 1989, 199 la), we estimated that $20-50 \%$ of red nucleus neurons produce burst discharges in response to a spinal stimulus whereas the others do not. Thus, many neurons do not receive intense synaptic input, and these may correlate with the unlabeled neurons observed in this study. The distribution of unlabeled neurons is particularly apparent in the cerebellar cortex (Figs. $2 B, 4,6$ ) since Purkinje cells are organized as a monolayer of neurons that are packed closely together. It is possible that the Purkinje cells that label heavily are projecting to nuclear and rubral cells that are unlabeled. This is an interesting hypothesis that will be important to explore in the future.

In summary, the patterns of dye uptake seen in the cerebellorubral circuit are consistent with the hypothesis that red nucleus burst discharge is sustained in part by positive feedback. The results also raise the possibility that various modulatory mechanisms could provide a finer control of the spatial patterns of activity within the cerebellorubral circuit. In the future, it will be important to explore these fine control mechanisms, since they may be very important in the generation of motor programs for controlling precise limb movements in space.

\section{Label present at other sites}

Sulforhodamine uptake was not confined to nuclei that, based on published anatomy, were expected to participate in the cerebellorubral circuit. Although efforts were made to limit the pathways stimulated by dissecting a single dorsal quadrant of the spinal cord, there is no guarantee that this would preserve only pathways that project to the cerebellorubral circuit. Some of the additional regions of uptake were quite variable between preparations, suggesting that their activation depended on pathways near the margins of the dissected spinal quadrant. The labeling of the vestibular complex and of the nucleus of the medial longitudinal fasciculus were in this highly variable category.

However, one region that showed quite consistent uptake was the raphe nucleus in the rostral brainstem (Fig. $4 D$ ). This region was labclcd in all but fivc cascs. Labcl was absent in three cases, since all synaptic transmission had been blocked by ionic sub- stitution in cases 13 and 14 , and since the spinal cord was not stimulated in case 12 . In another case, the antagonist APV had been administered, and the remaining case came from the initial experimental series (case 4). The remarkably consistent staining found for all but one case in which synaptic activity had not been blocked suggests that raphe neurons are strongly and quite directly activated by the spinal stimulus. These neurons may provide a major pathway for conducting excitation from the spinal cord to the red nucleus.

The label found in the optic tectum was fairly reliable in its presence, although the spatial patterns were generally different in each preparation (compare Figs. $4 A, 5 A, 6 A, 7$ ). Usually the label was more lateral on the side ipsilateral to the spinal stimulus, but beyond this, the patterns were complex and diverse. It was also interesting that dye uptake in the tectum was not blocked by APV, nor was it blocked in one of the CNQX cases.

\section{Comparison of sulforhodamine with other activity markers}

Recently, there has been much interest in studying the spatial patterns of brain activity associatcd with sensory or motor functions. Although dynamical methods are available for studying how spatial patterns of neural activity evolve over time, most of them sacrifice the extent of tissue sampled, frequently limiting it to a single plane or even a single spot, in order to obtain reasonable temporal resolution (Grinvald, 1985). In contrast, there are several static methods, including the sulforhodamine method described here, that have good spatial resolution. In this section, we compare sulforhodamine with two alternative static activity markers that are often used to study the spatial distribution of brain activity, namely ${ }^{14} \mathrm{C}$-2-deoxyglucose (2$\mathrm{DG})$ and the expression of the c-fos gene.

The 2-DG method has been used extensively since its introduction by Sokoloff et al. (1977). It relies on the uptake of a nonmetabolizable form of ${ }^{14} \mathrm{C}$-labeled glucose that then becomes trapped within the cell. The degree of 2-DG label relates to the intensity of metabolic activity, which is particularly large at active synapses. The regions with high metabolic activity are subsequently visualized by autoradiography. The chief disadvantages of this method are its limited resolution, the long processing time required for autoradiography, and difficulty in distinguishing between synaptic excitation and inhibition, both of which can enhance metabolic activity and 2-DG uptake.

Despite these disadvantages, the 2-DG method is a mainstay that has been used successfully to study a rich variety of problems. As a relevant example, Batini et al. (1981) used 2-DG to label spatial patterns of activity in the olivocerebellar system of the cat stimulated pharmacologically with harmaline. Harmaline induces rhythmic, synchronous discharges in the inferior olive and cerebellum. The patterns of 2-DG uptake in treated animals were enhanced in the inferior olive, cerebellar cortex, red nucleus, lateral reticular nucleus, nucleus reticularis tegmenti pontis, and the basal ganglia. The cercbellar nuclei showed a high level of activity in both control and the harmaline-treated group. It is clear that there is substantial similarity in the anatomical areas labeled in these experiments and those reported for sulforhodamine. In comparing the two methods, the main disadvantages of sulforhodamine are the present lack of a protocol for in vivo studies and the fact that it is a new, and therefore relatively untried, method. The main advantages are the easier and faster processing steps for sulforhodamine and its superior spatial resolution.

Expression of the fos protooncogene is significantly enhanced 
by many extracellular stimuli (Curran, 1988). Neurons in which this induction occurs can then be stained using immunohistochemistry for the Fos protein (Sharp et al., 1989b). Fos expression, in contrast to 2-DG, provides an excellent cellular resolution, although the label is contained exclusively in the cell nucleus since Fos is a nuclear regulatory protein. Correspondingly, for visualizing cell morphology, sulforhodamine is superior to both Fos and 2-DG. Soma, dendrites, and terminal fields are clearly stained by the sulforhodamine.

As with other markers, the spatial patterns of Fos labeling may be complex. Comparisons between Fos and 2-DG labeling demonstrate many similarities as well as substantial differences (Sagar et al., 1988; Sharp et al., 1989b). For example, similarly located patches of cerebellar cortex are labeled by the two techniques in response to electrical stimulation of the rat sensorimotor cortex. However, the labeling seen near the site of cortical stimulation in the contralateral cortex and in the striatum are substantially different. While 2-DG labeling is clearly related to metabolic activity, the range of stimuli producing alterations of Fos expression are not known. Electrical or synaptic activity seems not to be necessary, since local injections of NGF, or small lesions, in the cerebral cortex produce diffuse unilateral Fos expression throughout much of the cerebral hemisphere (Sharp ct al., 1989a). On the other hand, water deprivation leads to Fos expression in regions of hypothalamus known to respond to this stimulus, whereas these regions do not label with 2-DG (Sagar et al., 1988). The fact that there are many unknown steps between synaptic or electrical activity and Fos expression makes it difficult to be confident of the relationship to neural activity. The range of controls described here for sulforhodamine uptake have not yet been conducted for Fos expression.

These various considerations suggest sulforhodamine as a promising substance for marking the spatial distribution of activity in the nervous system. However, confidence in this dye can only be built up progressively by studying its labeling patterns in other neural systems. Along these lines, we have started to use sulforhodamine to label the neural correlate of the eyeblink reflex (Houk and Keifer, 1991). This reflex was evoked in the abducens nerve of the in vitro brainstem-cerebellum preparation by electrical stimulation of the trigeminal nerve (Keifer and Houk, 1990). Preparations showing strong abducens nerve reflexes demonstrated dye uptake in the trigeminal, accessory abducens, and principal abducens nuclei, and also in the reticular formation. Among these sites, only the reticular formation was stained in the present experiments with spinal cord stimulation. Furthermore, spinal stimulation stained the lateral cerebellar and red nucleus, sites that never stained with trigeminal nerve stimulation.

\section{Conclusions}

In this article, we have presented a variety of evidence suggesting that sulforhodamine 101 labels sites of neural activity, and we have utilized this dye to label neural circuits engaged in motor pattern generation in the in vitro brainstem-cerebellum of the turtle. The motor pattern studied here is the burst discharge in the red nucleus that is evoked by electrical stimulation of the spinal cord. The present observations support the hypothesis by Kcifcr and Houk (1991a) that recurrent excitation in the cerebellorubral circuits is an important factor in sustaining burst discharge. Hence, these circuits in the turtle may function as reverberatory loops as was proposed for the cat by Tsukahara et al. (1983). These results with sulforhodamine are significant because positive feedback is an emergent property of the circuit as a whole. Such a mechanism operating in the physiology of the nervous system would be difficult to demonstrate otherwise without recording from multiple sites simultaneously. Further physiological studies are needed to elucidate the cellular and circuit mechanisms underlying burst discharge of rubral neurons. However, microelectrode placements can now be guided by the patterns of label observed with sulforhodamine. These data may also support a more general theory of sensorimotor integration proposed by Houk $(1987,1989)$ suggesting that positive feedback between the cerebellum, motor cortex, and the red nucleus functions as a fundamental driving force for the generation of central motor programs controlling limb movement.

\section{References}

Bangma GC, Ten Donkelaar HJ (1982) Afferent connections of the cerebellum in various types of reptiles. J Comp Neurol 207:255-273. Bangma GC, Ten Donkelaar HJ, Dederen PJW, De Boer-van Huizen R (1984) Cerebellar efferents in the lizard Varanus exanthematicus. II. Projections of the cerebellar nuclei. J Comp Neurol 230:218-230.

Batini C, Buisseret-Demas C, Conrath-Verrier M (1981) Harmalineinduced tremor. I. Regional metabolic activity as revealed by $\left[{ }^{14} \mathrm{C}\right]$ 2-deoxyglucose in cat. Exp Brain Res 42:371-382.

Berry MS, Pentreath VW (1976) Criteria for distinguishing between monosynaptic and polysynaptic transmission. Brain Res 105:1-20.

Besterman JM, Airhart JA, Woodworth RC, Low RB (1981) Exocytosis of pinocytosed fluid in cultured cells: kinetic evidence for rapid turnover and compartmentation. J Cell Biol 91:716-727.

Borges LF, Elliot PJ, Gill R, Iversen SD, Iversen LL (1985) Selective extraction of small and large molecules from the cerebrospinal fluid by Purkinje neurons. Science 228:346-348.

Chan CY, Nicholson C (1986) Modulation by applied electric fields of Purkinje and stellate cell activity in the isolated turtle cerebellum. J Physiol (Lond) 371:89-114.

Cheney PD, Mewes K, Fetz EE (1988) Encoding of motor parameters by corticomotoneuronal (CM) and rubromotoneuronal (RM) cells producing possible facilitation of forelimb muscles in the behaving monkey. Behav Brain Res 28:181-191.

Curran T (1988) The fos oncogene. In: The oncogene handbook (Reddy EP, ed), pp 76-98. Amsterdam: Elsevier.

Ebbesson SOE (1969) Brain stem afferents from the spinal cord in a sample of reptilian and amphibian species. Ann NY Acad Sci 167: 80-101.

Ghez C, Kubota K (1977) Activity of red nucleus neurons with a skilled forelimb movement in the cat. Brain Res 129:383-388.

Gibson AR, Houk JC, Kohlerman NJ (1985a) Magnocellular red nucleus activity during different types of limb movement in the macaque monkey. J Physiol (Lond) 358:527-549.

Gibson AR, Houk JC, Kohlerman NJ (1985b) Relation between red nucleus discharge and movement parameters in trained macaque monkeys. J Physiol (Lond) 358:551-570.

Grillner S, Brodin L, Sigvardt K, Dale N (1986) On the spinal network generating locomotion in lamprey: transmitters, membrane properties and circuitry. In: Neurobiology of vertebrate locomotion (Grillner S, Stein PSG, Stuart DG, Forssberg H, Herman RM, eds), pp 335-352. London: Macmillan.

Grinvald A (1985) Real-time optical mapping of neuronal activity: from single growth cones to the intact mammalian brain. Annu Rev Neurosci 8:263-305.

Heuser JE, Reese TS (1973) Evidence for recycling of synaptic vesicle membrane during transmitter release at the frog neuromuscular junction. J Cell Biol 57:315-344.

Houk JC (1987) Model of the cerebellum as an array of adjustable pattern generators. In: Cerebellum and neuronal plasticity (Glickstein M, Yeo C, Stein J, eds), pp 249-260. New York: Plenum.

Houk JC (1989) Cooperative control of limb movements by the motor cortex, brainstem and cerebellum. In: Models of brain function (Cotterill RMJ, ed), pp 309-324. Cambridge: Cambridge UP.

Houk JC, Keifer J (1991) Visualization of the turtle eye-blink reflex arc with the activity-dependent dye sulforhodamine. Soc Neurosci Abstr 17:337. 
Houk JC, Keifer J, Vyas D (1989) Activity patterns in the cerebellorubrospinal pathway of the in vitro turtle hindbrain revealed with activity-dependent uptake of fluorescent dye. Soc Neurosci Abstr 15: 612.

Jahnsen H (1986) Electrophysiological characteristics of neurones in the guinea-pig deep cerebellar nuclei in vitro. J Physiol (Lond) 372 $129-147$.

Keifer J, Houk JC (1989) An in vitro preparation for studying motor pattern generation in the cerebellorubrospinal circuit of the turtle. Neurosci Lett 97:123-128.

Keifer J, Houk JC (1990) Conditioned response learning in the in vitro turtle brainstem-cerebellum. Soc Neurosci Abstr 16:763.

Keifer J, Houk JC (1991a) Rolc of excitatory amino acids in mediating burst discharge of red nucleus neurons in the in vitro turtle brainstemcerebellum. J Neurophysiol 65:454-467.

Keifer J, Houk JC (1991b) A slow EPSP contributes to burst discharge of turtle red nucleus neurons revealed by intracellular recording in vitro. Soc Neurosci Abstr 17:1024.

Keifer J, Vyas D, Houk JC, Berrebi AS, Mugnaini E (1992) Evidence for GABAergic interneurons in the red nucleus of the pond turtle. Synapse 11:197-213.

Kriegstein AR, Avilla JG, Blanton MG (1988) Distribution of increased synaptic activity during focal and generalized epileptiform activity revealed by presynaptic uptake of fluorescent dyes. Soc Neurosci Abstr 14:471.

Kubota M, Nakamura M, Tsukahara N (1985) Ionic conductance associated with electrical activity of guinca-pig red nuclcus ncuroncs in vitro. J Physiol (Lond) 362:161-171.

Kunzle H (1983) Supraspinal cell populations projecting to the cerebellar cortex in the turtle (Pseudemys scripta elegans). Exp Brain Res 49:1-12.

Kunzle H, Woodson W (1982) Mesodiencephalic and other target regions of ascending spinal projections in the turtle, Pseudemys scripta elegans. J Comp Neurol 212:349-364.

Lichtman JW, Wilkinson RS, Rich MM (1985) Multiple innervation of tonic endplates revealed by activity-dependent uptake of fluorescent probes. Nature 314:357-359.

Lichtman JW, Sunderland WJ, Wilkinson RS (1989) High-resolution imaging of synaptic structure with a simple confocal microscope. New Biol 1:75-82.

Llinas R, Muhlethaler M (1988) An electrophysiological study of the in vitro, perfused brain stem-cerebellum of adult guinea-pig. J Physiol (Lond) 404:215-240.

Lutz PL, Rosenthal M, Sick TJ (1985) Living without oxygen: turtle brain as a model of anaerobic metabolism. Mol Physiol 8:41 1-425.

Marcus LC (1981) Veterinary biology and medicine of captive amphibians and reptiles. Philadelphia: Lea and Febiger.

O'Donovan MJ (1989) Motor activity in the isolated spinal cord of the chick embryo: synaptic drive and firing pattern of single motoneurons. J Neurosci 9:943-958.
Roberts A, Soffe SR, Dale N (1986) Spinal interneurones and swimming in frog embryos. In: Neurobiology of vertebrate locomotion (Grillner S, Stein PSG, Stuart DG, Forssberg H, Herman RM, eds), pp 279-306. London: Macmillan.

Robinson FR, Houk JC, Gibson AR (1987) Limb specific connections of the cat magnocellular red nucleus. J Comp Neurol 257:553-577.

Sagar SM, Sharp FR, Curran T (1988) Expression of c-fos protein: metabolic mapping at the cellular level. Science 240:1328-1331.

Sarrafizadeh R, Keifer J, Houk JC (1989) Naturally and electrically evoked burst activity in the red nucleus of anesthetized turtles. Soc Neurosci Abstr 15:390.

Sarrafizadeh R, Keifer J, Houk JC (1990) Suppression of somatosensory input to the red nucleus during movement in the anesthetized turtle. Soc Neurosci Abstr 16:112.

Sharp FR, Gonzalez MF, Hisanaga K, Mobley WC, Sagar SM (1989a) Induction of the c-fos gene product in rat forebrain following cortical lesions and NGF injections. Neurosci Lett 100:117-122.

Sharp FR, Gonzalez MF, Sharp JW, Sagar SM (1989b) c-fos expression and $\left({ }^{14} \mathrm{C}\right) 2$-deoxyglucose uptake in the caudal cerebellum of the rat during motor/sensory cortex stimulation. J Comp Neurol 284:621636.

Sladeczek F, Recasens M, Bockaert J (1988) A new mechanism for glutamate receptor action: phosphoinositide hydrolysis. Trends Neurosci 11:545-549.

Sokoloff L, Reivich M, Kennedy CH, DesRosiers MH, Patlak CS, Pettigrew KD, Sakurada O, Shinohara M (1977) The $\left({ }^{14} \mathrm{C}\right)$ deoxyglucose method for the measurement of local cerebral glucose utilization: theory, procedure, and normal values in the conscious and anesthetized albino rat. J Neurochem 28:897-916.

Swanson JA, Yirinec BD, Silverstein SC (1985) Phorbol esters and horseradish peroxidase stimulate pinocytosis and redirect the flow of pinocytosed fluid in macrophages. J Cell Biol 100:851-859.

Ten Donkelaar HJ (1976) Descending pathways from the brain stem to the spinal cord in some reptiles. II. Course and site of termination. J Comp Neurol 167:443-464.

Ten Donkelaar HJ (1982) Organization of descending pathways to the spinal cord in amphibians and reptiles. Prog Brain Res 57:25-67.

Tsukahara N, Bando T, Kiyohara T (1973) The propertics of the reverberating circuit in the brain. In: Neuroendocrine control (Yagi K, Yoshida S, eds), pp 3-26. Tokyo: Tokyo UP.

Tsukahara N, Bando T, Murakami F, Oda Y (1983) Properties of cerebello-precerebellar reverberating circuits. Brain Res 274:249-259.

Wang Y, Goren MB (1987) Differential and sequential delivery of fluorescent lysosomal probes into phagosomes in mouse peritoneal macrophages. J Cell Biol 104:1749-1754.

Wilcox M, Franceschini N (1984) Illumination induces dye incorporation in photoreceptor cells. Science 225:851-854. 